\title{
Involvement of WNT Signaling in the Regulation of Gestational Age-Dependent Umbilical Cord-Derived Mesenchymal Stem Cell Proliferation
}

\author{
Sota Iwatani, ${ }^{1}$ Akemi Shono, ${ }^{1}$ Makiko Yoshida, ${ }^{2}$ Keiji Yamana, ${ }^{1}$ Khin Kyae Mon Thwin, ${ }^{1}$ \\ Jumpei Kuroda, ${ }^{3}$ Daisuke Kurokawa, ${ }^{1}$ Tsubasa Koda, ${ }^{4}$ Kosuke Nishida, ${ }^{1}$ Toshihiko Ikuta, \\ Kazumichi Fujioka, ${ }^{1}$ Masami Mizobuchi, ${ }^{5}$ Mariko Taniguchi-Ikeda, ${ }^{1}$ Ichiro Morioka, ${ }^{1}$ \\ Kazumoto Iijima, ${ }^{1}$ and Noriyuki Nishimura ${ }^{1}$ \\ ${ }^{1}$ Department of Pediatrics, Kobe University Graduate School of Medicine, Kobe 6500017, Japan \\ ${ }^{2}$ Department of Pathology, Kobe Children's Hospital, Kobe 6500047, Japan \\ ${ }^{3}$ Department of Neonatology, Tokyo Metropolitan Children's Medical Center, Fuchu 1838561, Japan \\ ${ }^{4}$ Department of Pediatrics, Hyogo College of Medicine, Nishinomiya 6638181, Japan \\ ${ }^{5}$ Department of Developmental Pediatrics, Shizuoka Children's Hospital, Shizuoka 4208660, Japan \\ Correspondence should be addressed to Noriyuki Nishimura; nnishi@med.kobe-u.ac.jp
}

Received 17 April 2017; Revised 22 June 2017; Accepted 4 July 2017; Published 12 September 2017

Academic Editor: Zhaohui Ye

Copyright (c) 2017 Sota Iwatani et al. This is an open access article distributed under the Creative Commons Attribution License, which permits unrestricted use, distribution, and reproduction in any medium, provided the original work is properly cited.

\begin{abstract}
Mesenchymal stem cells (MSCs) are a heterogeneous cell population that is isolated initially from the bone marrow (BM) and subsequently almost all tissues including umbilical cord (UC). UC-derived MSCs (UC-MSCs) have attracted an increasing attention as a source for cell therapy against various degenerative diseases due to their vigorous proliferation and differentiation. Although the cell proliferation and differentiation of BM-derived MSCs is known to decline with age, the functional difference between preterm and term UC-MSCs is poorly characterized. In the present study, we isolated UC-MSCs from 23 infants delivered at 22-40 weeks of gestation and analyzed their gene expression and cell proliferation. Microarray analysis revealed that global gene expression in preterm UC-MSCs was distinct from term UC-MSCs. WNT signaling impacts on a variety of tissue stem cell proliferation and differentiation, and its pathway genes were enriched in differentially expressed genes between preterm and term UC-MSCs. Cell proliferation of preterm UC-MSCs was significantly enhanced compared to term UC-MSCs and counteracted by WNT signaling inhibitor XAV939. Furthermore, WNT2B expression in UC-MSCs showed a significant negative correlation with gestational age (GA). These results suggest that WNT signaling is involved in the regulation of GAdependent UC-MSC proliferation.
\end{abstract}

\section{Introduction}

Mesenchymal stem cells (MSCs) are a heterogeneous cell population that has a potential to proliferate and differentiate into trilineage mesenchymal cells: adipocytes, osteocytes, and chondrocytes. MSCs were initially isolated and characterized from the bone marrow $(\mathrm{BM})[1,2]$ and subsequently derived from almost all tissues including adipose tissue (AT), synovium, skin, dental pulp, umbilical cord blood (UCB), placenta, and umbilical cord (UC) [3]. Due to the ability to home to sites of injury, undergo differentiation, suppress immune responses, and modulate angiogenesis, MSCs are paid an increasing attention as a source for cell therapy against various degenerative diseases. Currently, MSCs from different sources have been tested in clinical studies for treatment of graft-versus-host disease, myocardial infarction, cerebral infarction, and so on $[4,5]$.

Although BM is the most well-characterized source of MSCs, it has certain limitations with the invasive BM aspiration and the decline in MSC proliferation and 
differentiation capacity with age. In contrast, fetal MSCs obtained from UCB, placenta, and UC have advantages with the noninvasive sampling during newborn delivery and the vigorous proliferation and differentiation capacity for cell therapy [6,7]. Especially, human UC starts to develop at 4-8 weeks of gestation, continues to grow until $50-60 \mathrm{~cm}$ in length, and is usually discarded as medical waste after newborn delivery. Taken together, UC-derived MSCs (UC-MSCs) will become a promising source for cell therapy $[8,9]$.

Various genes and signaling pathways are known to regulate MSC proliferation and differentiation. WNT signaling serves as a key regulator that influences various stages of embryonic development as well as tissue homeostasis in adulthood [10]. It affects the proliferation, selfrenewal, and differentiation of various tissue stem cells and controls the various tissue renewal and regeneration in response to disease, trauma, and ageing [11]. WNT ligands, which comprise a family of 19 members in human, are evolutionally conserved, are lipid modified, and secreted glycoproteins. They can activate either $\beta$-catenindependent (canonical) or $\beta$-catenin-independent (noncanonical) pathways by acting on transmembrane receptor FZD and its coreceptors LRP5/LRP6. The canonical pathway inhibits the $\beta$-catenin destruction complex, associates the transcriptional coactivator $\beta$-catenin with the transcriptional factor complex TCF/LEF, and induces WNT target gene transcription. The noncanonical pathway is independent of $\beta$-catenin and mainly associates with $\mathrm{Ca}^{2+}$-dependent and JNK-dependent signaling pathways, which can impact on cell migration, cell polarity, and cytoskeletal organization. The molecular events occurring these noncanonical pathways are far less defined than the canonical pathway [12].

Early studies showed the profound impacts of WNT signaling on a variety of tissue stem cell proliferation and differentiation [13-15]. In MSCs, both stimulatory and inhibitory roles for WNT signaling in cell proliferation and differentiation into trilineage mesenchymal cells were documented [16]. The adipogenic differentiation of AT-derived MSCs (AT-MSCs) was inhibited by WNT signaling activation [17]. The potential of WNT signaling on osteogenic differentiation of MSCs was controversial, with both stimulatory and inhibitory effects being reported $[18,19]$. An inhibitory effect of WNT signaling on chondrogenic differentiation was demonstrated in AT-MSCs [20].

Although UC can be obtained from a wide range of gestational age (GA) newborn as a result of preterm, term, and postterm delivery, their functional differences are poorly characterized [21]. An understanding of the molecular mechanisms controlling UC-MSC proliferation and differentiation is crucial to determining the drivers and effectors of the functional difference between different GA UC-MSCs as well as the most suitable use of UC-MSCs for cell therapy against degenerative diseases. In the present study, we isolated UC-MSCs from 23 infants delivered at 22-40 weeks of gestation and analyzed their gene expression and cell proliferation.

\section{Materials and Methods}

2.1. Patients and Samples. Human UCs were obtained from 23 infants delivered at 22-40 weeks of gestation with parental written consent. This study was approved by the Ethics Committee at Kobe University Graduate School of Medicine (approval number 1370) and Hyogo Prefectural Kobe Children's Hospital (approval numbers 24-25) and conducted in accordance with the approved guidelines.

2.2. Preparation of UC-MSC. The umbilical cord (2-3g weight) was collected, cut into $2-3 \mathrm{~mm}$ pieces, enzymatically dissociated with Liberase DH Research Grade (Roche, Mannheim, Germany) in PBS for $45-60 \mathrm{~min}$ at $37^{\circ} \mathrm{C}$ followed by the addition of $10 \%$ fetal bovine serum (FBS; Sigma, St. Louis, MO) to inhibit enzyme activity, and filtered through a $100 \mu \mathrm{m}$ cell strainer (BD Bioscience, Bedford, MA). The resulting cells derived from all compartments of the umbilical cord (whole UC) were cultured at $37^{\circ} \mathrm{C}\left(5 \% \mathrm{CO}_{2}\right.$ and 95\% air) in MEM- $\alpha$ (Wako Pure Chemical, Osaka, Japan) containing $10 \%$ FBS and $1 \%$ antibiotic-antimycotic solution (Invitrogen, Carlsbad, CA) until confluent primary cultures were established. The cells were then disassociated with trypsin-EDTA (Wako Pure Chemical), and the trypsinized cells were seeded into fresh dishes and passaged to confluence. Serial passaging was carried out until the tenth passage. The cells at fifth to eighth passages were used in the present experiments.

2.3. Cell Surface Marker Analysis. UC-MSCs were dissociated with $0.25 \%$ trypsin-EDTA for 10 minutes, washed with PBS and suspended at $\sim 1 \times 10^{6}$ cells $/ \mathrm{ml}$ in FCM buffer containing $1 \times$ PBS, 2 mM EDTA, and 10\% Block Ace (Dainippon Pharmaceutical, Osaka, Japan). The cells were incubated with phycoeryhrin- (PE-) conjugated mouse primary antibodies against CD14, CD19, CD34, CD45, CD73, CD90, CD105, or HLA-DR (BD Bioscience, Franklin Lakes, NJ) for 45 min on ice, washed with PBS, incubated with Fixable Viability Stain 450 (BD Bioscience) for $15 \mathrm{~min}$ at room temperature, washed with PBS, and filtered through a $70 \mu \mathrm{m}$ cell strainer (BD Bioscience). PE-conjugated mouse IgG1 k, IgG2a k, or IgG2b k isotype control (BD Bioscience) was used as a negative control for each primary antibody. Flow cytometric analysis was performed using FACSAria III carrying a triple laser (BD Bioscience) and FACSDiva software (BD Bioscience).

2.4. Cell Differentiation. To verify the multipotency of UCMSCs, the cells were induced to differentiate into the adipogenic, osteogenic, and chondrogenic lineages. Adipogenic differentiation was induced in STEMPRO adipogenesis differentiation medium (Invitrogen) for 2-3 weeks and stained, and the differentiation was investigated by staining lipid vesicles with Oil Red O (Sigma). Osteogenic differentiation was induced in STEMPRO osteogenesis differentiation medium (Invitrogen) or STK-3 (DS Pharma Biomedical, Osaka, Japan) for 1-2 weeks, and the differentiation was examined by staining with Arizarin Red S (Sigma) reacting to calcium cation. Chondrogenic differentiation was induced by forming cell aggregates in micromass culture in STEMPRO chondorogenesis differentiation medium (Invitrogen) for 
1 week, and the differentiation was assessed by staining anionic glycoconjugates with Toluidine Blue (Sigma). Cell images were acquired using a BZ-X700 microscope (Keyence, Osaka, Japan).

2.5. RNA Extraction. Total RNA from UC-MSCs and fibroblasts was extracted with a TRIZOL Plus RNA purification kit (Life Technologies) according to the manufacturer's instructions. RNA integrity was evaluated by Agilent 2100 Bioanalyzer (Agilent Technologies, Santa Clara, CA) using RNA 6000 nanokit (Agilent Technologies) according to the manufacturer's instructions.

2.6. Gene Expression Microarray Analysis. Total RNA from three term and five preterm UC-MSCs (Table 1) was subjected to global gene expression analysis using the Low Input Quick Amp Labeling Kit One-Color (Agilent Technologies) and SurePrint G3 Human Gene Expression v3 $8 \times 60 \mathrm{~K}$ Microarray Kit (Agilent Technologies) according to the manufacturer's instruction. Briefly, double-stranded cDNA was synthesized from $100 \mathrm{ng}$ of total RNA by AffinityScript-RT using T7 promoter-incorporated Oligo-dT primer. Cyanine 3- (Су3-) CTP-incorporated RNA (cRNA) was generated using the second strand cDNA as a template via an in vitro transcription reaction. The amplified cRNA was purified with the RNeasy mini kit (Qiagen, Valencia, CA) and quantified cRNA by the NanoDrop 2000 (Thermo Fisher Scientific, Waltham, MA). $600 \mathrm{ng}$ of Cy3-labeled cRNA was hybridized to the microarray slides at $65^{\circ} \mathrm{C}$ for $17 \mathrm{hr}$ with rotation at $10 \mathrm{rpm}$. After hybridization, the slides were washed and scanned by the SureScan (Agilent Technologies), the images were subsequently extracted using the Feature Extraction Software (Agilent Technologies). Extracted data with good QC metrics were normalized (percentile shift to the 75th percentile) and filtered by gene expression (20.0-100.0 percentile), flags for signals and error for CV in the GeneSpring GX (v 14.5) (Agilent Technologies). The processed data were subjected to statistical analysis (moderated $T$-test with Benjamini-Hochberg FDR), and the corrected $p$ value $<0.05$ was determined to be significant $(n=3-5)$. The following analyses were performed for further data interpretation: principal component analysis (PCA), clustering analysis, GO (gene ontology) analysis, and pathway analysis with curated datasets of WikiPathways (413 pathways) and KEGG (10 pathways). A gene-set list associated with human WNT signaling pathway (150 genes, 04310 from KEGG pathways) was obtained from a public database (https://www.stemformatics.org/).

2.7. Quantitative RT-PCR (RT-qPCR). cDNA was synthesized from $1 \mu \mathrm{g}$ of total RNA from UC-MSCs by using a QuantiTect reverse transcription kit (Qiagen). Real-time PCR analysis was performed with an ABI 7500 real-time PCR system (Applied Biosystems, Foster City, CA) using FastStart Universal SYBR Green master mix (Roche) with $0.5 \mu \mathrm{M}$ sense and antisense primers and cDNA (corresponding to $12.5 \mathrm{ng}$ total RNA) according to the manufacturer's instructions. Each cDNA was amplified with a precycling hold at $95^{\circ} \mathrm{C}$ for $10 \mathrm{~min}$, followed by 40 cycles at $95^{\circ} \mathrm{C}$ for
$15 \mathrm{sec}$ and $60^{\circ} \mathrm{C}$ for $60 \mathrm{sec}$, and one cycle at $95^{\circ} \mathrm{C}$ for $15 \mathrm{sec}$, $60^{\circ} \mathrm{C}$ for $60 \mathrm{sec}, 95^{\circ} \mathrm{C}$ for $15 \mathrm{sec}$, and $60^{\circ} \mathrm{C}$ for $15 \mathrm{sec}$. Relative expression of each transcript was calculated based on the $\Delta \Delta \mathrm{Ct}$ method using $\beta$-actin (ACTB) as an endogenous reference for normalization. Primer sequences for WNT2, WNT2B, WNT3A, WNT4, WNT5B, WNT6, SFRP1, and ACTB were shown in Table 2. All sample measurements were repeated at least three times, and the results were expressed as the mean $\pm \mathrm{SE}$.

2.8. Ki-67 Staining. Cell suspensions of UC-MSCs were centrifuged at $3000 \mathrm{rpm}$ for $5 \mathrm{~min}$, and two smears were immediately prepared. Slides were fixed in $95 \%$ ethanol for immunostaining or fixed in 20\% formalin and $80 \%$ methanol and stained with hematoxylin and eosin (H\&E), respectively. Immunostaining was performed with antibody against Ki-67 (Clone MIB-1, Dako, Santa Clara, CA) using Leica BondMax automation and Bond Polymer Refine detection kit (Leica Biosystems, Nussloch, Germany) according to manufacturer's instructions. IHC cytology protocol included primary antibody incubation for $15 \mathrm{~min}$, post primary for $8 \mathrm{~min}$, polymer for $8 \mathrm{~min}$, peroxide block for $5 \mathrm{~min}$, mixed $\mathrm{DAB}$ refine for $10 \mathrm{~min}$, and followed by $5 \mathrm{~min}$ hematoxylin counterstaining.

2.9. MTS Assay. UC-MSCs were seeded at the density of 12,000 cells/well in a 12-well plate, incubated in $1 \mathrm{ml}$ of MEM- $\alpha$ with $10 \%$ FBS in the presence or absence of $10 \mu \mathrm{M}$ XAV939 (Selleck Chemicals, Houston, TX) at $37^{\circ} \mathrm{C}(5 \%$ $\mathrm{CO}_{2}$ and $95 \%$ air) for 24,48 , or $72 \mathrm{~h}$. Cell proliferation was then determined by the CellTiter 96H AQueous One Solution Cell Proliferation Assay kit (Promega, Madison, WI, USA) according to the manufacturer's instruction. Briefly, $200 \mu \mathrm{l}$ of MTS reagent (a tetrazolium compound) was added into each well and incubated at $37^{\circ} \mathrm{C}\left(5 \% \mathrm{CO}_{2}\right.$ and $95 \%$ air $)$ for $4 \mathrm{~h}$. The absorbance at $490 \mathrm{~nm}$ was measured using an EnSpire Microplate Reader (Perkin Elmer, Poland, OR). All experiments were repeated at least three times, and the results were expressed as the mean \pm SE.

2.10. Statistical Analysis. Pearson's correlation coefficients were determined, and the Mann-Whitney $U$ test was used to compare two independent datasets, using Excel software (Microsoft, Redmond, WA) and Excel Statistics (Statcel 3; Social Survey Research Information, Tokyo, Japan). Differences were considered statistically significant for $p<0.05$.

\section{Results}

3.1. UC-MSCs Isolated from Infants Delivered at 22-40 Weeks of Gestation. We first obtained UCs from infants delivered at 22-40 weeks of gestation and then isolated the plasticadherent cells from these UCs (Table 1). The cells exhibited a spindle-like shape (Figure 1(a)). Their cell surface markers were positive for MSC signature markers CD73, CD90, and CD105 but negative for hematopoietic, macrophage, and endothelial markers CD14, CD19, CD34, CD45, and HLADR by flow cytometric analysis (Figure 1(b)). There were no statistically significant differences in the percentages of MSC signature marker-positive cells (CD73: 99.9 $\pm 0.1 \%$ 


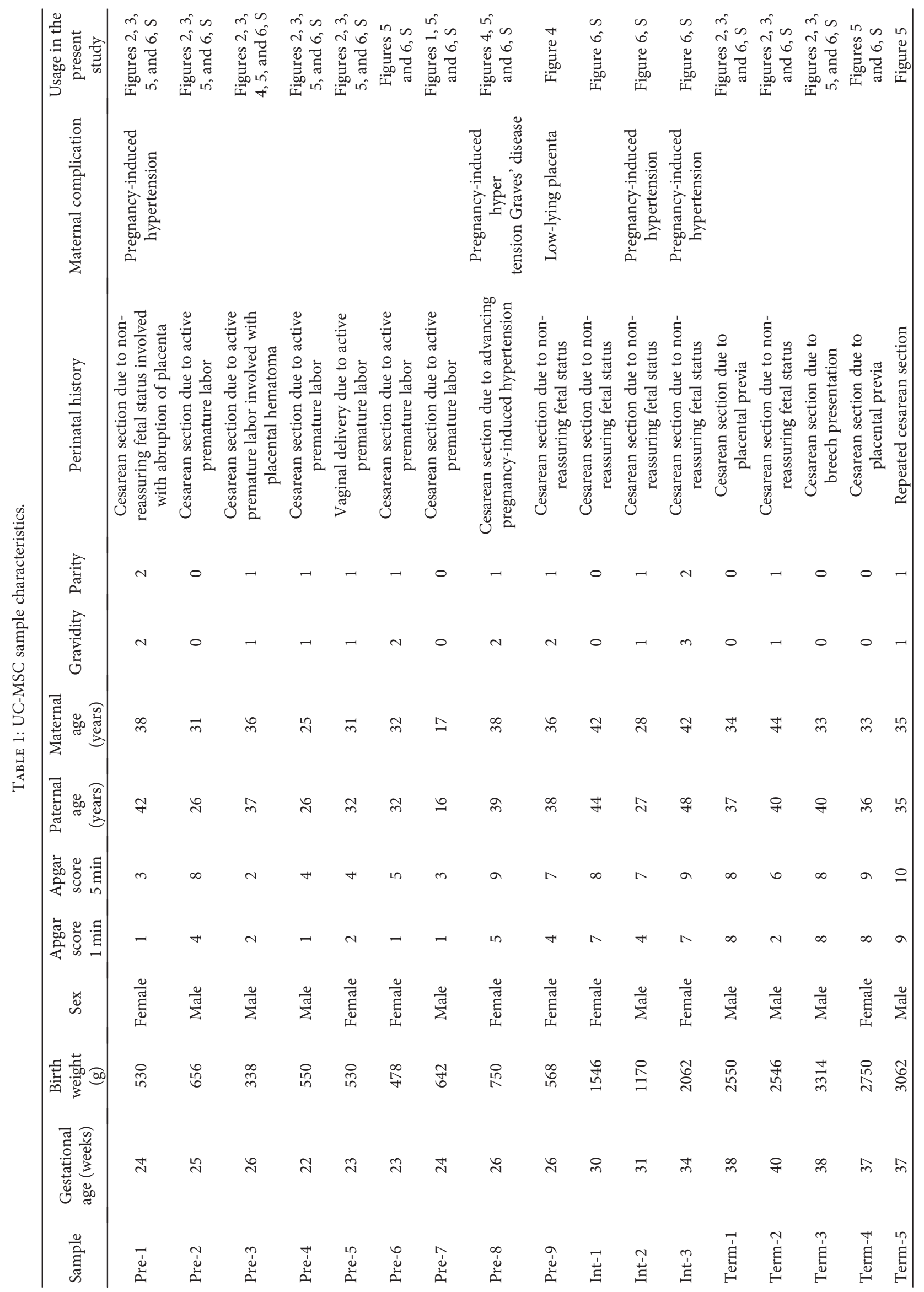




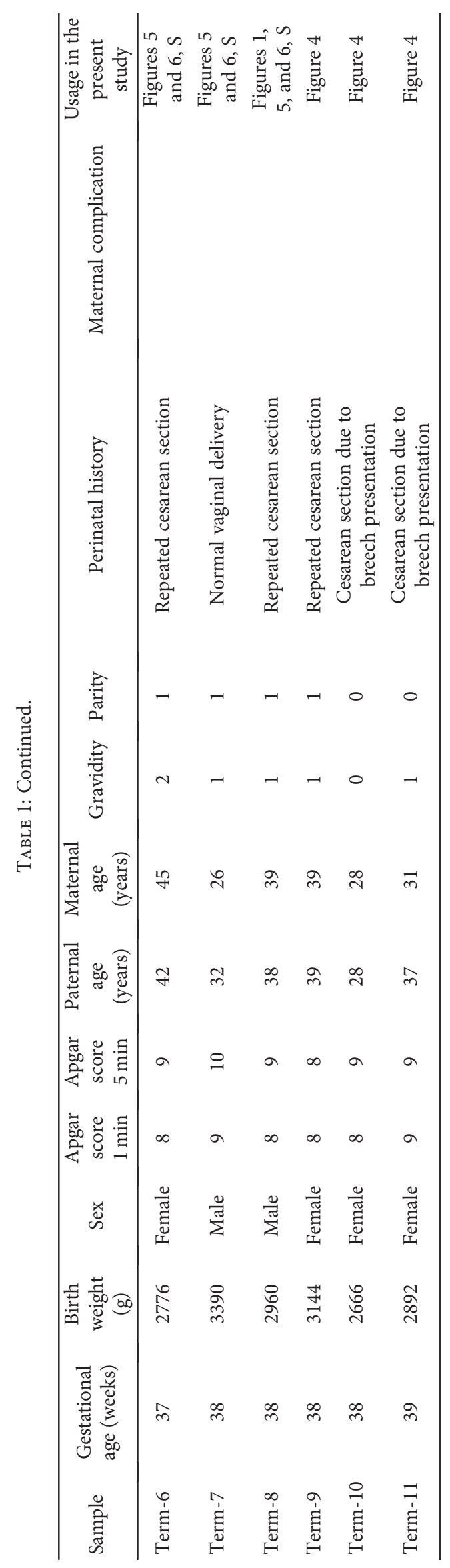


TABLE 2: Primers used for RT-qPCR.

\begin{tabular}{lcc}
\hline & Forward primer & Reverse primer \\
\hline WNT2 & tttggcagggtcctactcc & cctggtgatggcaaatacaa \\
$W N T 2 B$ & aacttacataataaccgctgtggtc & actcacgccatggcactt \\
$W N T 3 A$ & aactgcaccaccgtccac & aaggccgactccctggta \\
$W N T 4$ & gcagagccctcatgaacct & cacccgcatgtgtgtcag \\
WNT5B & gcgagaagactggaatcagg & cagagcagccgtgaacag \\
$W N T 6$ & agagtgccagttccagttcc & gaacacgaaggccgtctc \\
SFRP1 & gctggagcacgagaccat & tggcagttcttgttgagca \\
ACTB & ccaaccgcgagaagatga & ccagaggcgtacagggatag \\
\hline
\end{tabular}

and $99.6 \pm 0.4 \%, C D 90: 99.9 \pm 0.1 \%$ and $99.5 \pm 0.5 \%$, and CD105: $99.7 \pm 0.3 \%$ and $99.5 \pm 0.5 \%)$ between preterm and term UCs.

Under standard in vitro differentiation conditions, both preterm and term UC-MSCs were induced to differentiate into osteocytes, adipocytes, and chondrocytes (Figure 1(c)). Preterm UC-MSCs did not qualitatively differ from term UC-MSCs in their capacity to differentiate into trilineage mesenchymal cells. Taken together, the resulting cells fulfilled the criteria defined by the ISCT position paper [22] and were defined as UC-MSCs.

3.2. Differentially Expressed Genes between Preterm and Term UC-MSCs. To get an insight into the functional difference between preterm and term UC-MSCs, we extracted total RNA from five preterm and three term UC-MSCs (Table 1) and performed microarray analysis. Principal component analysis (PCA) for global gene expression revealed that preterm UC-MSC samples were clustered together and were separated from term UC-MSC samples (Figure 2(a)). In total, 5578 unique genes (4272 upregulated and 1306 downregulated) showed greater than twofold-expression changes between preterm and term UC-MSCs with a corrected $p$ value less than 0.05 (Figure 2(b), Supplementary Table S1 available online at https://doi.org/10.1155/2017/8749751). The pathway analysis of all differentially expressed genes identified significant enrichment of signaling pathways for immune/inflammatory reactions, cell-cell/cell-extracellular matrix interactions, glucose/lipid metabolism, and cell proliferation and differentiation (Table 3). Among these signaling pathways, we focused WNT signaling pathway that was previously implicated in the regulation of MSC proliferation and differentiation. Noticeably, 32/150 of WNT signaling pathway genes were overlapped with differentially expressed genes between preterm and term UC-MSCs (Figures 2(c) and 2(d)).

We then confirmed a subset of these WNT signaling pathway gene expressions by RT-qPCR using cDNA from the same five preterm and three term UC-MSCs as a template. A subset included secreted WNT ligands and modulators: WNT2, WNT2B, WNT3A, WNT4, WNT5B, WNT6, and SFRP1. Consistent with microarray analysis, upregulated WNT2, WNT2B, WNT3A, WNT4, and WNT6 showed increased expression in preterm UC-MSCs compared to term UC-MSCs by RT-qPCR (Table 4, Figure 3). Decreased expression of downregulated WNT5B and SFRP1 was also detected by RT-qPCR (Table 4, Figure 3). Collectively, these results suggested that WNT signaling pathway gene expression in preterm UC-MSCs was distinct from term UC-MSCs.

3.3. Cell Proliferation of Preterm and Term UC-MSCs. To examine the function of WNT signaling pathway genes in preterm and term UC-MSCs, we isolated UC-MSCs from nine preterm (22-26 weeks of gestation) and nine term (37-39 weeks of gestation) infants (Table 1) and analyzed their cell proliferation. We first evaluated the expression of Ki-67, a marker of proliferating cells expressed in all active phases of the cell cycle (G1, S, G2, and M), by immunocytochemistry [23]. The percentages of Ki-67-positive cells were markedly increased in preterm UC-MSCs as compared to term UC-MSCs, albeit not statistically significant (Figure 4).

We then analyzed cell proliferation of preterm and term UC-MSCs by MTS assay. Although both preterm and term UC-MSCs showed vigorous proliferation, the proliferation rate of preterm UC-MSCs measured at $72 \mathrm{~h}$ was significantly faster than term UC-MSCs (Figure 5(a)). Next, we examined the effect of WNT signaling inhibition on the growth of preterm and term UC-MSCs using a small molecule XAV939. XAV939 is a potent inhibitor of Tankyrase1 and Tankyrase2, and this inhibition stabilizes Axin1 and Axin2, the concentration-limiting component of the WNT pathway transcription factor $\beta$-catenin destruction complex. Increased levels of Axin 1 and Axin 2 stimulate $\beta$-catenin degradation and thereby inhibit $\beta$-catenin-mediated transcription [24]. Treatment of preterm UC-MSCs with $10 \mu \mathrm{M}$ XAV939 resulted in significant inhibition of cell proliferation (Figure 5(b)). Term UC-MSC proliferation was also reduced by $10 \mu \mathrm{M}$ XAV939, but there was no statistical significance (Figure 5(c)). These results suggest that WNT signaling is involved in the enhanced cell proliferation of preterm UC-MSCs compared to term UC-MSCs.

3.4. Gestational Age-Dependent Expression of WNT Signaling Pathway Genes. We further analyzed WNT2, WNT2B, WNT3A, WNT4, WNT5B, WNT6, and SFRP1 expressions in UC-MSCs isolated from other 10 infants delivered at 2240 weeks of gestation by RT-qPCR. Expression of these WNT signaling pathway genes tended to decrease or increase with gestational age. Among them, WNT2B expression showed a statistically significant negative correlation with gestational age (Figure 6, Supplementary Figure S1).

\section{Discussion}

In the present study, we isolated UC-MSCs from 23 infants delivered at 22-40 weeks of gestation and obtained the following findings. (1) Global gene expression in preterm UC-MSCs was distinct from term UC-MSCs. (2) WNT signaling pathway genes were enriched in differentially expressed genes between preterm and term UC-MSCs. (3) Preterm UC-MSC proliferation was faster than term UCMSCs. (4) WNT signaling inhibitor XAV939 significantly inhibited the cell proliferation of preterm but not term 

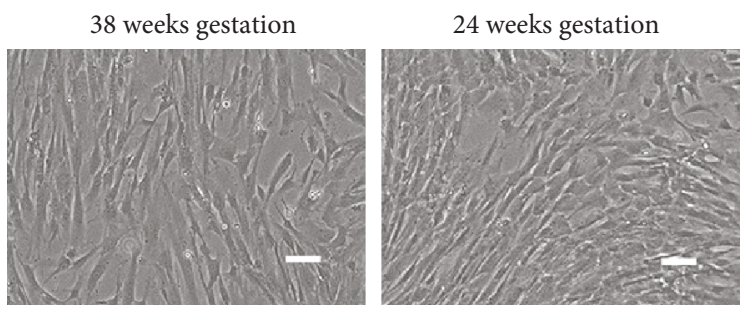

(a)
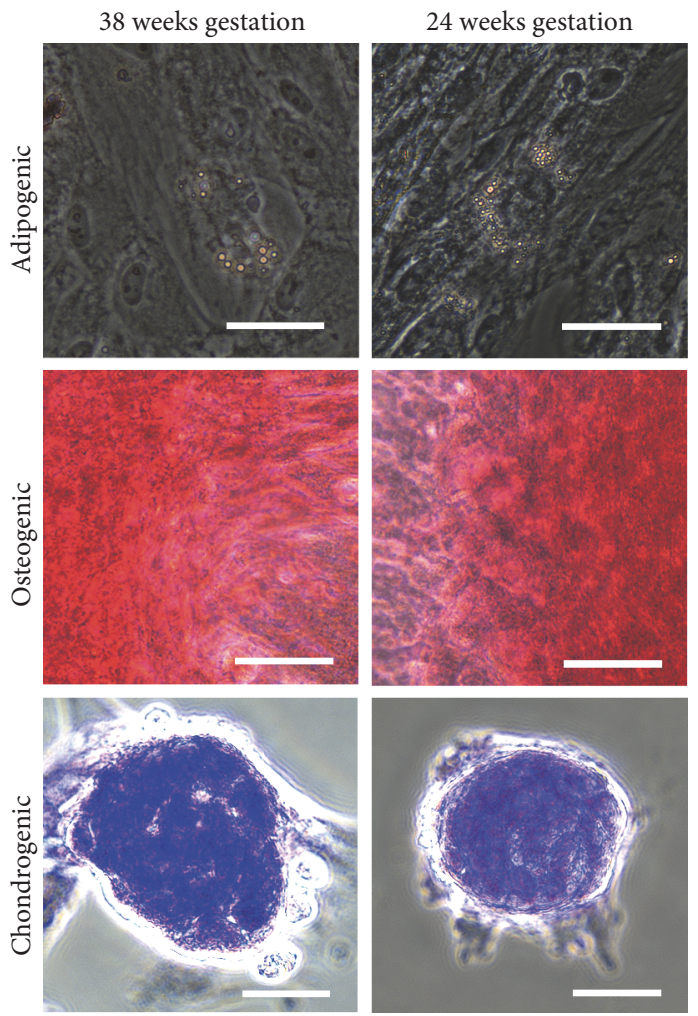

(c)
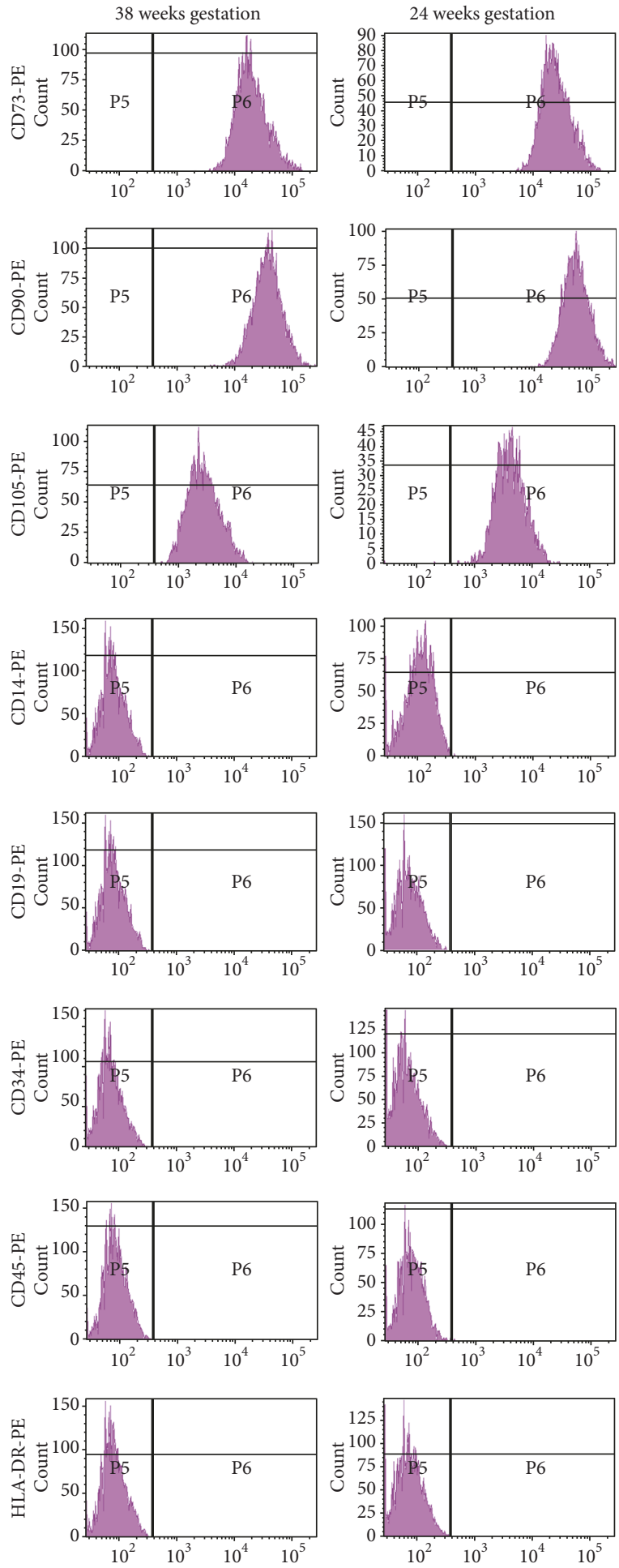

(b)

FIGURE 1: Characterization of UC-MSCs from term and preterm infants. (a) UC-MSCs from preterm (24 weeks of gestation, preterm UCMSCs) and term (38 weeks of gestation, term UC-MSCs) newborns at passage numbers 6 to 7 were examined by phase-contrast microscopy. The images shown are representative of three independent experiments. Scale bars show $100 \mu \mathrm{m}$. (b) Preterm and term UCMSCs were analyzed by flow cytometer using antibodies against MSC markers (CD14, CD19, CD34, CD45, CD73, CD90, CD105, and HLA-DR) defined by ISCT [22]. The histograms shown are representative of three independent experiments. (c) Preterm and term UCMSCs were differentiated into adipocyte as visualized by Oil Red O and into osteocyte as visualized by Alizarin Red S and chondrocyte as visualized by Toluidine Blue. The images shown are representative of three independent experiments. Scale bars represent $50 \mu \mathrm{m}$. 


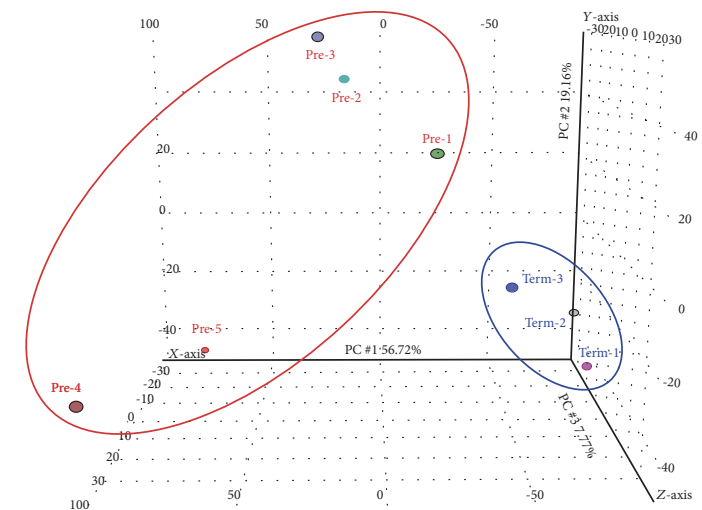

(a)

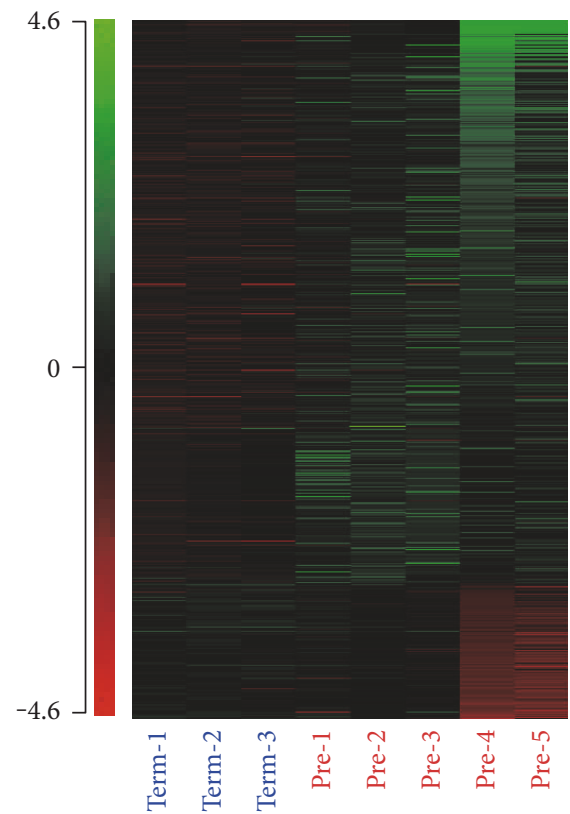

(b)
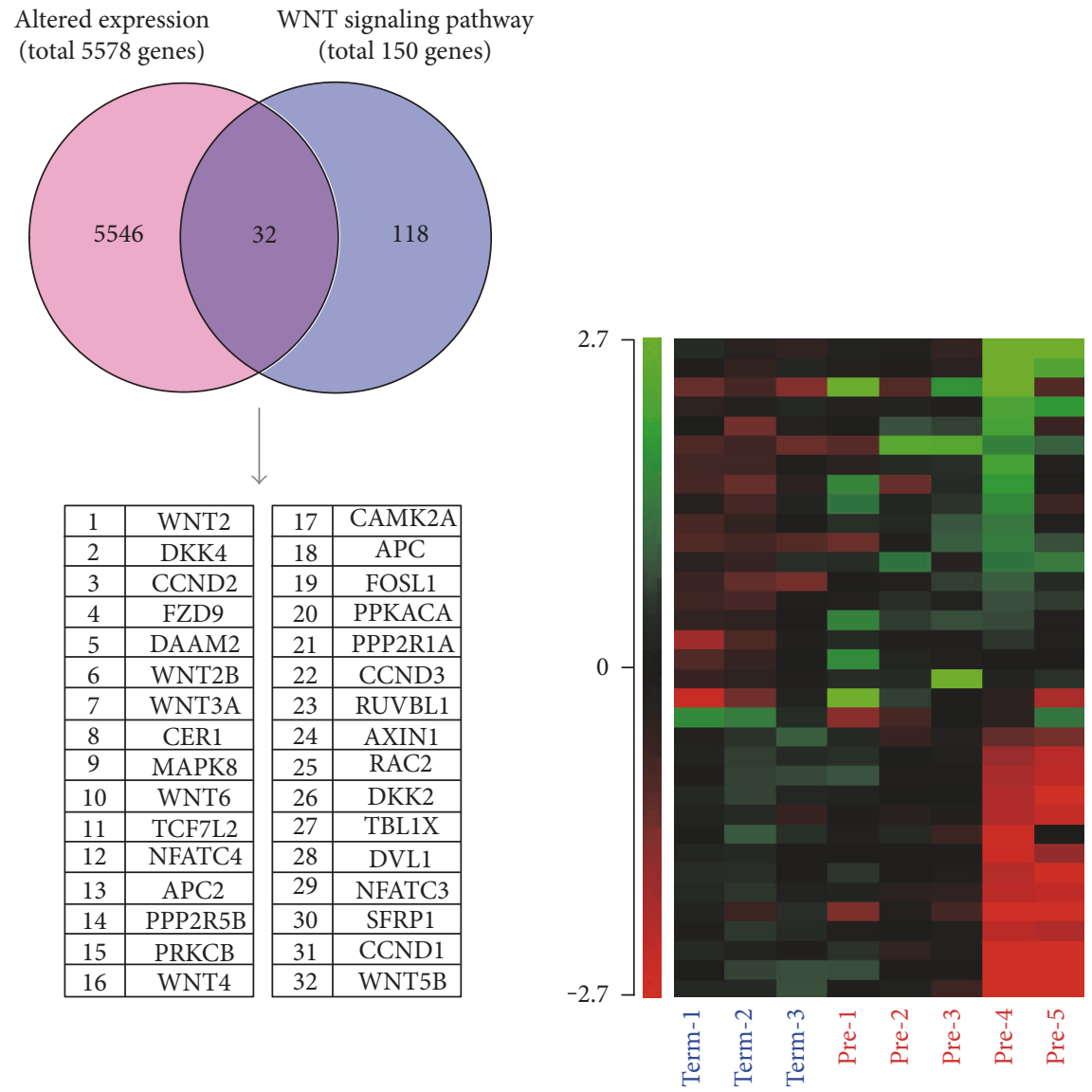

(c)

(d)

Figure 2: Gene expression microarray analysis of preterm and term UC-MSCs. (a) PCA mapping of gene expression profile for preterm (pre-1-5) and term (term-1-3) UC-MSCs. (b) Heat map of 5578 differentially expressed gene with greater than twofold changes in preterm UC-MSCs as compared to term UC-MSCs at a corrected $p$ value less than 0.05 . Green color refers to low levels of gene expression and red color to high levels. (c) Pie chart of altered expression genes and WNT signaling pathway genes. The list shows overlapped 32 genes. (d) Heat map of 32 genes extracted from (c). Green color refers to low levels of gene expression and red color to high levels. 


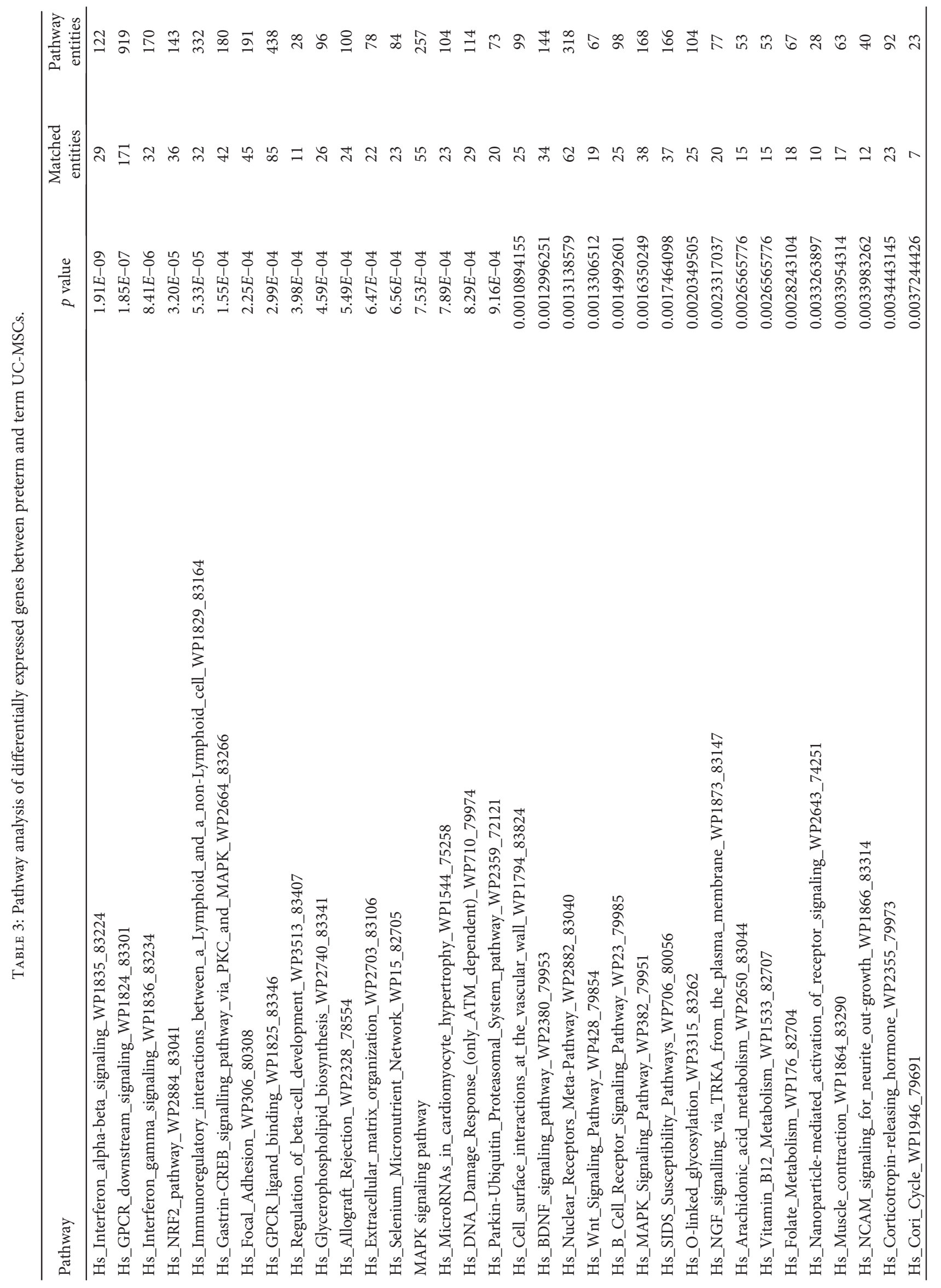




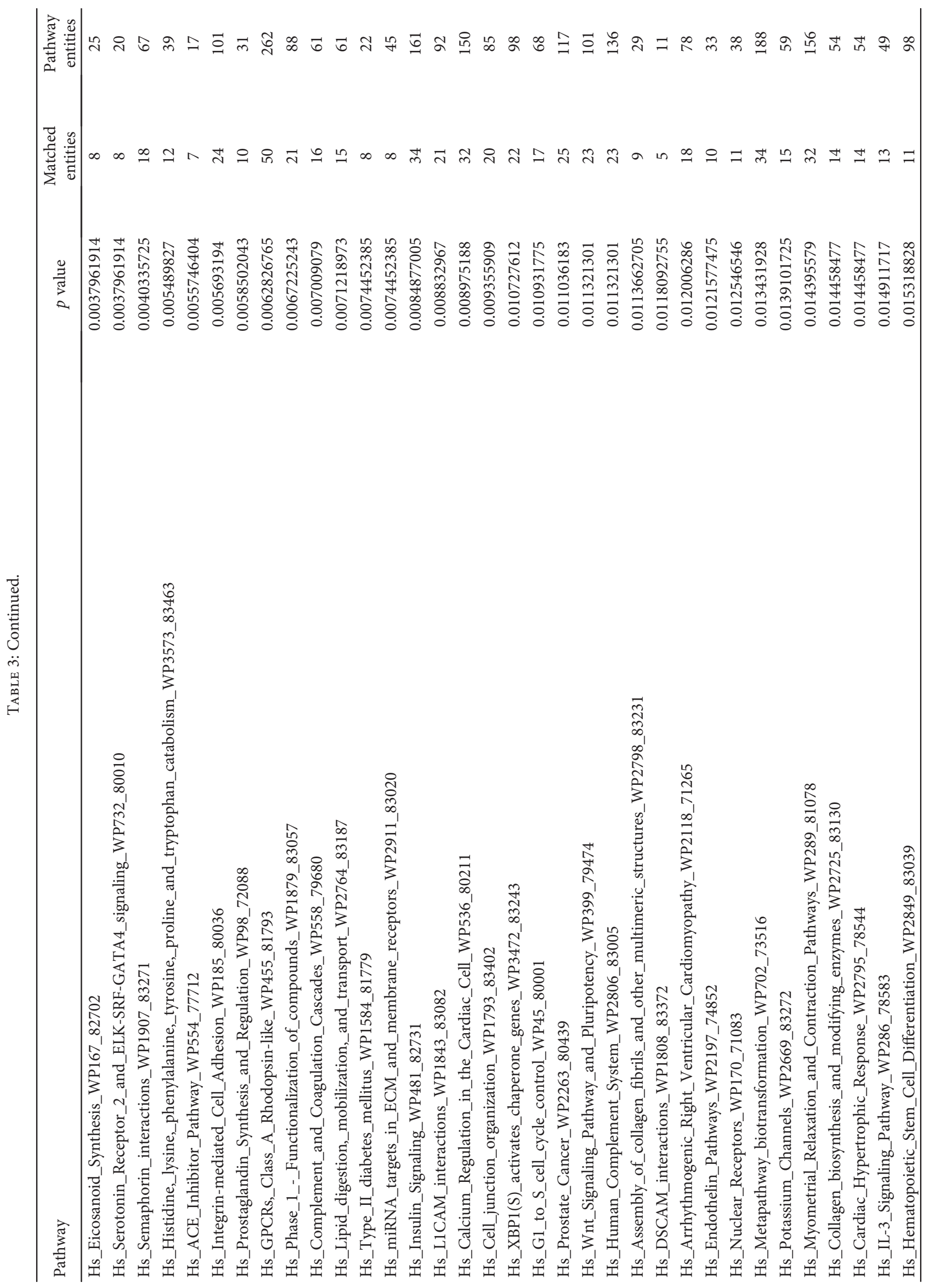




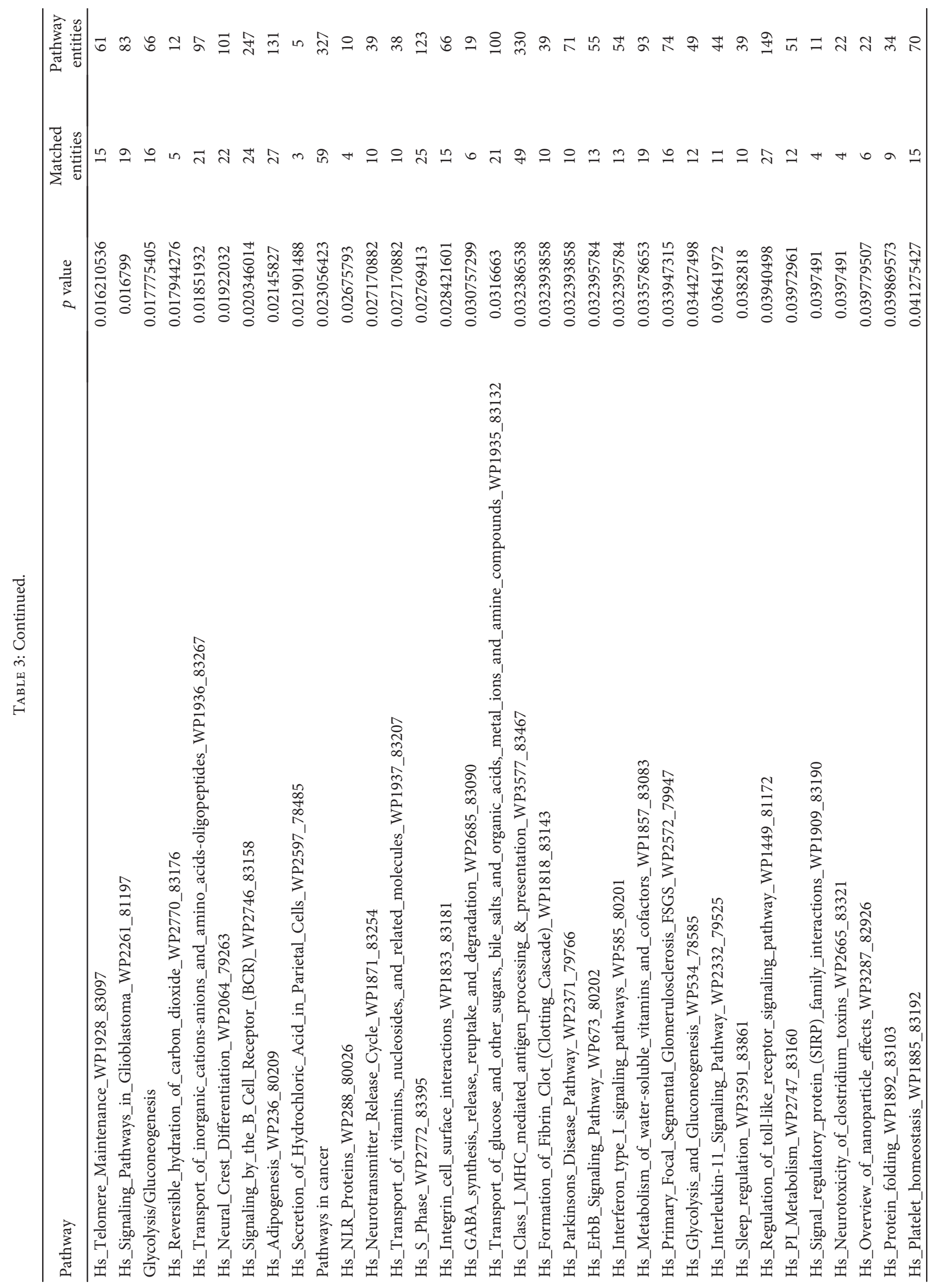




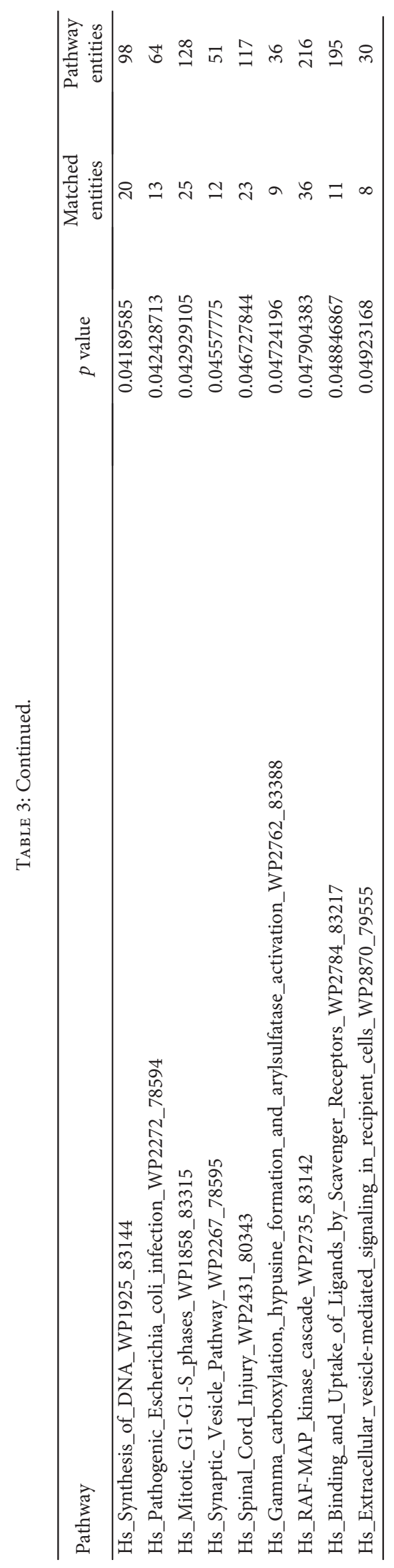


TABLE 4: Differentially expressed WNT pathway genes between preterm and term UC-MSCs.

\begin{tabular}{|c|c|c|}
\hline Gene & FC (pre versus term) & $p$ (Corr) \\
\hline \multicolumn{3}{|l|}{ Ligands } \\
\hline WNT2 & 4.07917 & 0.01631 \\
\hline WNT2B & 2.64791 & 0.02698 \\
\hline WNT3A & 2.56017 & 0.02584 \\
\hline WNT6 & 2.35531 & 0.00938 \\
\hline WNT4 & 2.11623 & 0.00510 \\
\hline WNT5B & -3.93533 & 0.01317 \\
\hline \multicolumn{3}{|l|}{ Receptors } \\
\hline FZD9 & 3.47820 & 0.04035 \\
\hline TCF7L2 & 2.29222 & 0.01187 \\
\hline \multicolumn{3}{|c|}{ Extracellular modulators } \\
\hline DKK4 & 3.79029 & 0.01143 \\
\hline$D K K 2$ & -2.65327 & 0.01720 \\
\hline SFRP1 & -3.13336 & 0.00621 \\
\hline \multicolumn{3}{|c|}{ Intracellular signaling molecules } \\
\hline CCND2 & 3.49892 & 0.02933 \\
\hline DAAM2 & 2.71289 & 0.00467 \\
\hline CER1 & 2.40160 & 0.03446 \\
\hline MAPK8 & 2.36091 & 0.00501 \\
\hline NFATC4 & 2.25687 & 0.01089 \\
\hline$A P C 2$ & 2.21774 & 0.04864 \\
\hline PPP2R5B & 2.20956 & 0.00501 \\
\hline$P R K C B$ & 2.11780 & 0.00504 \\
\hline CAMK2A & 2.03443 & 0.02825 \\
\hline$A P C$ & -2.01861 & 0.00578 \\
\hline FOSL1 & -2.06872 & 0.03346 \\
\hline PRKACA & -2.16403 & 0.02705 \\
\hline PPP2R1A & -2.19719 & 0.04119 \\
\hline CCND3 & -2.20727 & 0.01748 \\
\hline$R U V B L 1$ & -2.24427 & 0.02646 \\
\hline AXIN1 & -2.47832 & 0.02670 \\
\hline$R A C 2$ & -2.51880 & 0.00902 \\
\hline TBL1X & -2.73244 & 0.01261 \\
\hline DVL1 & -2.77299 & 0.00726 \\
\hline NFATC3 & -2.82561 & 0.02238 \\
\hline CCND1 & -3.28580 & 0.03436 \\
\hline
\end{tabular}

UC-MSCs. (5) WNT2B expression in UC-MSCs showed a significant negative correlation with GA.

MSCs are isolated from a variety of tissues and result in so heterogeneous population of cells, and not all of them express the same phenotypic markers. In the case of BMMSCs, younger donor-derived BM-MSCs showed greater proliferative and differentiative potential than older counterparts and may have more potential for cell therapy $[25,26]$. Although fetal MSCs could be isolated from newborns delivered at a wide range of GA as a result of preterm, term, and postterm delivery, their GA-dependent function remained poorly characterized $[8,9]$. With regard to UCB-MSCs, the

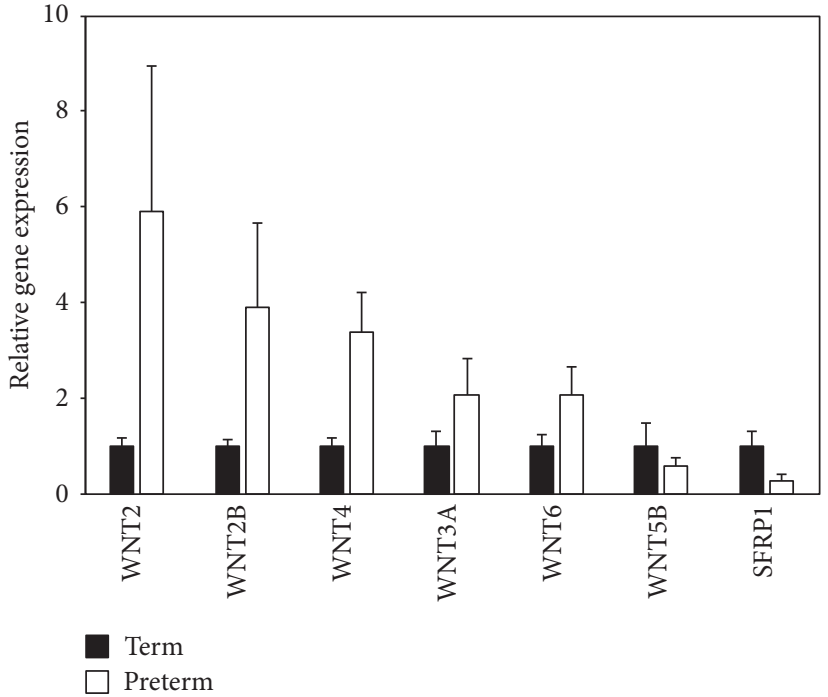

FIGURE 3: WNT signaling pathway gene expression in preterm and term UC-MSCs. The relative expression of WNT2, WNT2B, WNT3A, WNT4, WNT6, WNT5B, and SFRP1 mRNA in preterm $(n=3)$ and term $(n=5)$ UC-MSCs was analyzed by RT-qPCR. The mean of term UC-MSCs was set as 1 . The results shown are the mean $\pm S E$.
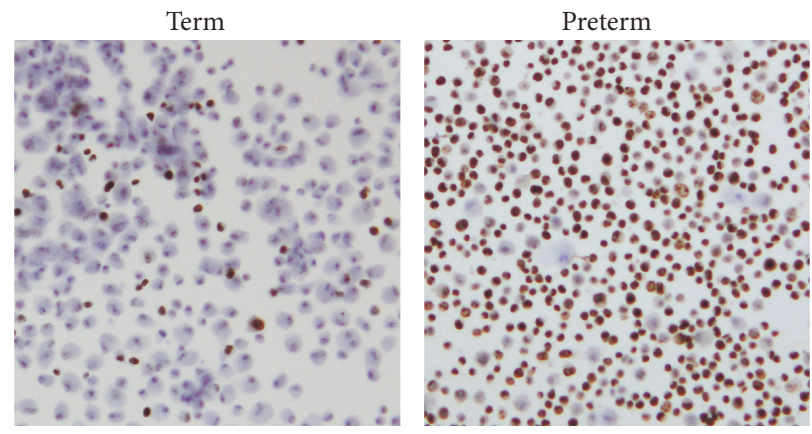

(a)

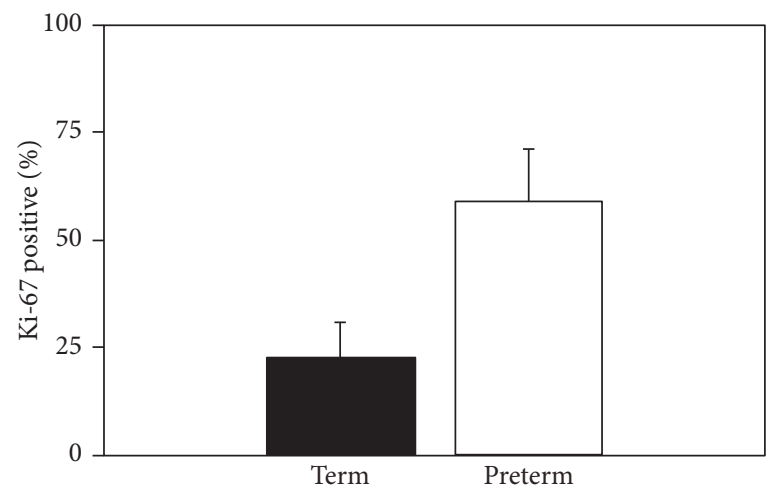

(b)

FIgURE 4: Ki-67 staining of preterm and term UC-MSCs. (a) Smears of preterm $(n=3)$ and term $(n=3)$ UC-MSCs were prepared, immunostained with anti-Ki-67 antibody, and counterstained with hematoxylin. The images shown are representative of three independent experiments. (b) The percentage of Ki-67 positive was determined by manually counting 1000 cells and expressed as the mean \pm SE. 


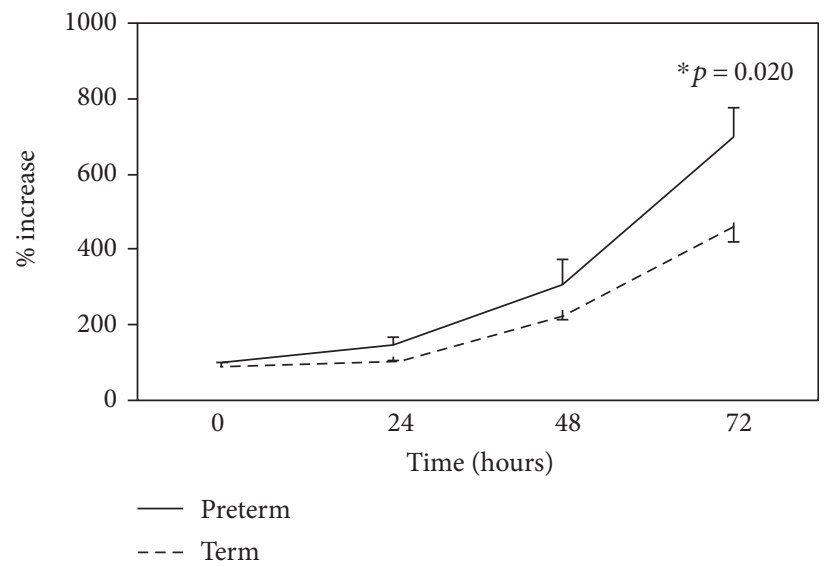

(a)

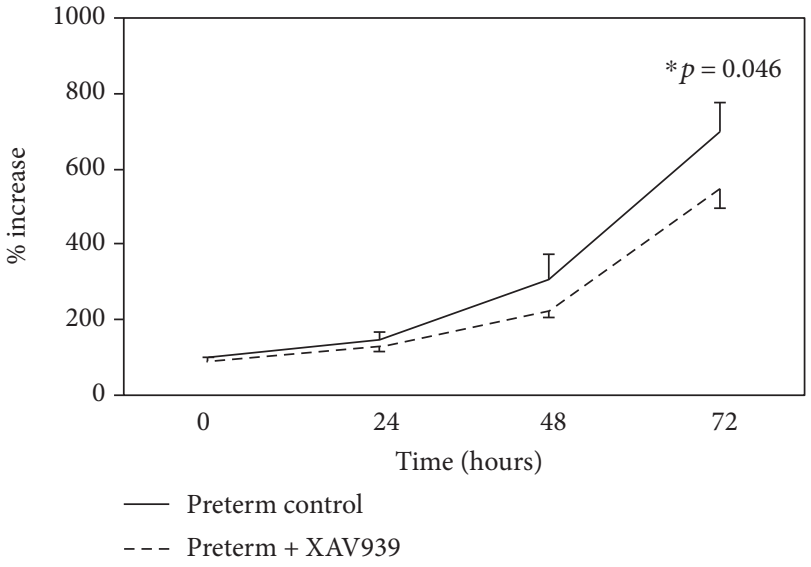

(b)

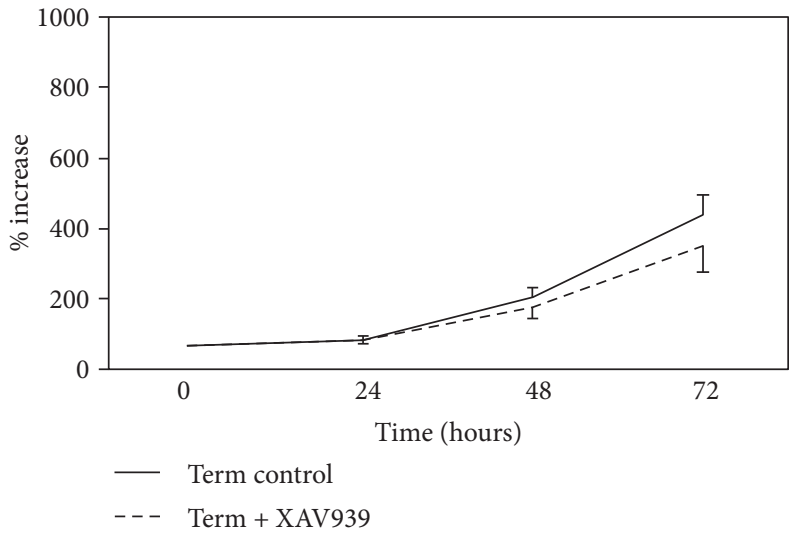

(c)

Figure 5: Cell proliferation of preterm and term UC-MSCs. Preterm $(n=8)$ and term $(n=6)$ UC-MSCs were cultured in the absence or presence of XAV939 for 24, 48, and $72 \mathrm{~h}$. Their cell proliferation was determined by MTS assay and expressed as the percent increase. The results shown are the mean \pm SE of (a) preterm and term UC-MSCs, (b) preterm UC-MSCs \pm XAV939, and (c) term UC-MSCs \pm XAV939.

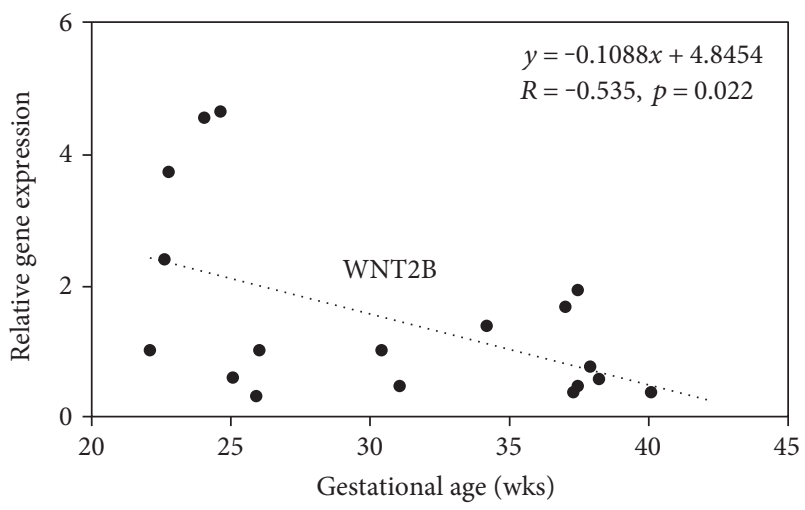

FIGURE 6: Gestational age-dependent expression of WNT signaling pathway genes. The relative expression of WNT2B mRNA in UCMSCs isolated from 18 infants delivered at 22-40 weeks of gestation was analyzed by RT-qPCR. The mean of all UC-MSCs was defined as 1 .

MSC population in UCB was significantly higher in preterm newborn compared to term newborn $[27,28]$. In the case of UC-MSCs, MSCs were isolated from different UC compartments including cord lining, perivascular region (PV), Wharton's jelly (WJ), and whole UC [29-31]. Preterm UCs were shown to contain more perivascular cells (PVCs), identical to MSCs, than term UCs [32]. Preterm PVCs/UC-MSCs isolated from fetuses aborted at 8-12 weeks of gestation were reported to exhibit a greater proliferative potential, a more efficient differentiation into chondrogenic and adipogenic cell lineages, and a differential gene expression profile compared to term PVCs/UC-MSCs isolated from newborns delivered at 37-40 weeks of gestation [33]. Although we isolated UC-MSCs from the whole UC and preterm newborns delivered at 22-26 weeks of gestation, the present study and others supported that proliferative capacity of UC-MSCs declined with GA.

Global gene expression analysis identified 5578 differentially expressed genes between preterm and term UC-MSCs (Figure 2(a), Table S1). The pathway analysis revealed significant enrichment of 111 signaling pathways (Table 3). Immune/inflammatory reaction-associated signaling pathways were top-ranked among the list (Table 3). The upregulation of interferon (IFN) signaling pathways in preterm UC-MSCs may be interpreted as the consequence of 
preterm delivery that has inherent fetal and/or maternal indications (Table 1, Table 3). Although cell cycle and senescence-associated secretory phenotype pathways were also expected to affect the growth rate and GA-dependent changes of UC-MSCs, these pathways were not included in the list (Table 3).

WNT signaling is a key regulator of stem cell functions in development, renewal, and regeneration of multiple tissues [13-15]. In the case of MSCs, mRNA expression of a subset of WNT signaling pathway genes including WNT2, WNT4, WNT5A, WNT11, WNT16, SFRP2, SFRP3, and SFRP4 was detected in BM-MSCs [34]. WNT2, WNT2B, WNT4, WNT5A, WNT5B, SFRP1, and SFRP4 were also highly expressed in AT-MSCs under hypoxic stress conditions [35]. Comparison of BM-MSCs with UC-MSCs revealed lower differentiation capacity toward osteocytes and adipocytes along with the downregulation of WNT3A, WNT5A, WNT5B, WNT7B, WNT8A, SFRP1, and SFRP4 in UCMSCs compared to BM-MSCs [36]. Consistent with these observations, the present study revealed a significant enrichment of WNT2, WNT2B, WNT3A, WNT4, WNT5B, WNT6, and SFRP1 in differentially expressed genes between preterm and term UC-MSCs (Figure 2(c)). Noticeably, WNT2, WNT2B, WNT4, WNT5B, WNT6, and SFRP1 were associated with a noncanonical WNT pathway, as opposed to only WNT3A with a canonical WNT pathway among these WNT ligands and modulators in UC-MSCs [12]. In contrast, the enhanced cell proliferation of preterm UC-MSCs was abolished by XAV939, which selectively decreased $\beta$-catenin expression through Tankyrase 1 and Tankyrase 2 inhibition and increased Axin1 and Axin2 expression (Figure 5) [24]. Accumulating evidence indicates that noncanonical WNT signaling can inhibit canonical WNT signaling [37, 38] and that activation of either canonical or noncanonical WNT signaling is highly dependent on the cell type and on specific receptors expressed by the cells $[39,40]$. Further understanding of how WNT signaling pathway controls the GA-dependent proliferation of UC-MSC will be crucial to develop UC-MSCbased cell therapy.

In summary, preterm UC-MSC proliferation is significantly faster than term UC-MSCs, and WNT signaling is involved in the regulation of this GA-dependent proliferation of UC-MSCs.

\section{Conflicts of Interest}

The authors declare that there is no conflict of interest regarding the publication of this paper.

\section{Acknowledgments}

This work was supported by Grants-in-Aid for Scientific Research (C) (Grant no. 25461644) and Young Scientists (B) (Grant no. 26860845) of JSPS KAKENHI. The authors thank Drs. Shohei Ohyama, Sachiyo Fukushima, Oshi Tokuda, Kaori Maeyama, and Miwako Nagasaka for collecting the umbilical cord samples.

\section{References}

[1] A. J. Friedenstein, K. V. Petrakova, A. I. Kurolesova, and G. P. Frolova, "Heterotopic of bone marrow. Analysis of precursor cells for osteogenic and hematopoietic tissues," Transplantation, vol. 6, no. 2, pp. 230-247, 1968.

[2] A. I. Caplan, "Mesenchymal stem cells," Journal of Orthopaedic Research, vol. 9, no. 5, pp. 641-650, 1991.

[3] M. Crisan, S. Yap, L. Casteilla et al., "A perivascular origin for mesenchymal stem cells in multiple human organs," Cell Stem Cell, vol. 3, no. 3, pp. 301-313, 2008.

[4] P. Bianco, P. G. Robey, and P. J. Simmons, "Mesenchymal stem cells: revisiting history, concepts, and assays," Cell Stem Cell, vol. 2, no. 4, pp. 313-319, 2008.

[5] P. Bianco, “"Mesenchymal” stem cells," Annual Review of Cell and Developmental Biology, vol. 30, no. 1, pp. 677-704, 2014.

[6] D. Baksh, R. Yao, and R. S. Tuan, "Comparison of proliferative and multilineage differentiation potential of human mesenchymal stem cells derived from umbilical cord and bone marrow," Stem Cells, vol. 25, no. 6, pp. 1384-1392, 2007.

[7] S. Manochantr, Y. U-pratya, P. Kheolamai et al., "Immunosuppressive properties of mesenchymal stromal cells derived from amnion, placenta, wharton's jelly and umbilical cord," Internal Medicine Journal, vol. 43, no. 4, pp. 430-439, 2013.

[8] D.-C. Ding, Y.-H. Chang, W.-C. Shyu, and S.-Z. Lin, "Human umbilical cord mesenchymal stem cells: a new era for stem cell therapy," Cell Transplantation, vol. 24, no. 3, pp. 339-347, 2015.

[9] I. Arutyunyan, A. Elchaninov, A. Makarov, and T. Fatkhudinov, "Umbilical cord as prospective source for mesenchymal stem cell-based therapy," Stem Cells International, vol. 2016, Article ID 6901286, 17 pages, 2016.

[10] H. Clevers and R. Nusse, "Wnt/ $\beta$-catenin signaling and disease," Cell, vol. 149, no. 6, pp. 1192-1205, 2012.

[11] H. Clevers, K. M. Loh, and R. Nusse, "Stem cell signaling. An integral program for tissue renewal and regeneration: Wnt signaling and stem cell control," Science, vol. 346, no. 6205, article 1248012, 2014.

[12] H. A. Baarsma, M. Königshoff, and R. Gosens, “The Wnt signaling pathway from ligand secretion to gene transcription: molecular mechanisms and pharmacological targets," Pharmacology \& Therapeutics, vol. 138, no. 1, pp. 66-83, 2013.

[13] V. Korinek, N. Barker, P. Moerer et al., "Depletion of epithelial stem-cell compartments in the small intestine of mice lacking tcf-4," Nature Genetics, vol. 19, no. 4, pp. 379-383, 1998.

[14] U. Gat, R. DasGupta, L. Degenstein, and E. Fuchs, "De novo hair follicle morphogenesis and hair tumors in mice expressing a truncated beta-catenin in skin," Cell, vol. 95, no. 5, pp. 605614, 1998.

[15] R. DasGupta and E. Fuchs, "Multiple roles for activated lef/tcf transcription complexes during hair follicle development and differentiation," Development, vol. 126, no. 20, pp. 45574568, 1999.

[16] M. Visweswaran, S. Pohl, F. Arfuso et al., "Multi-lineage differentiation of mesenchymal stem cells - to Wnt, or not Wnt," The International Journal of Biochemistry \& Cell Biology, vol. 68, pp. 139-147, 2015.

[17] J. R. Park, J.-W. Jung, Y.-S. Lee, and K.-S. Kang, "The roles of Wnt antagonists dkk1 and sfrp4 during adipogenesis of human adipose tissue-derived mesenchymal stem cells," Cell Proliferation, vol. 41, no. 6, pp. 859-874, 2008. 
[18] C. A. Gregory, W. G. Gunn, E. Reyes et al., "How Wnt signaling affects bone repair by mesenchymal stem cells from the bone marrow," Annals of the New York Academy of Sciences, vol. 1049, no. 1, pp. 97-106, 2005.

[19] J. de Boer, R. Siddappa, C. Gaspar, A. van Apeldoorn, R. Fodde, and C. van Blitterswijk, "Wnt signaling inhibits osteogenic differentiation of human mesenchymal stem cells," Bone, vol. 34, no. 5, pp. 818-826, 2004.

[20] S. Luo, Q. Shi, Z. Zha et al., "Inactivation of Wnt/ $\beta$-catenin signaling in human adipose-derived stem cells is necessary for chondrogenic differentiation and maintenance," Biomedicine \& Pharmacotherapy, vol. 67, no. 8, pp. 819-824, 2013.

[21] D. Zhu, E. M. Wallace, and R. Lim, "Cell-based therapies for the preterm infant," Cytotherapy, vol. 16, no. 12, pp. 16141628, 2014.

[22] M. Dominici, K. Le Blanc, I. Mueller et al., "Minimal criteria for defining multipotent mesenchymal stromal cells. The international society for cellular therapy position statement," Cytotherapy, vol. 8, no. 4, pp. 315-317, 2006.

[23] J. Gerdes, H. Lemke, H. Baisch, H. H. Wacker, U. Schwab, and H. Stein, "Cell cycle analysis of a cell proliferation-associated human nuclear antigen defined by the monoclonal antibody ki-67," Journal of Immunology, vol. 133, no. 4, pp. 1710$1715,1984$.

[24] S.-M. A. Huang, Y. M. Mishina, S. Liu et al., "Tankyrase inhibition stabilizes axin and antagonizes Wnt signalling," Nature, vol. 461, no. 7264, pp. 614-620, 2009.

[25] K. Mareschi, I. Ferrero, D. Rustichelli et al., "Expansion of mesenchymal stem cells isolated from pediatric and adult donor bone marrow," Journal of Cellular Biochemistry, vol. 97, no. 4, pp. 744-754, 2006.

[26] D. M. Choumerianou, G. Martimianaki, E. Stiakaki, L. Kalmanti, M. Kalmanti, and H. Dimitriou, "Comparative study of stemness characteristics of mesenchymal cells from bone marrow of children and adults," Cytotherapy, vol. 12, no. 7, pp. 881-887, 2010.

[27] M. J. Javed, L. E. Mead, D. Prater et al., "Endothelial colony forming cells and mesenchymal stem cells are enriched at different gestational ages in human umbilical cord blood," Pediatric Research, vol. 64, no. 1, pp. 68-73, 2008.

[28] A. Jain, N. Mathur, M. Jeevashankar et al., "Does mesenchymal stem cell population in umbilical cord blood vary at different gestational periods?," Indian Journal of Pediatrics, vol. 80, no. 5, pp. 375-379, 2013.

[29] M. T. Conconi and R. Di Liddo, "Phenotype and differentiation potential of stromal populations obtained from various zones of human umbilical cord: an overview," The Open Tissue Engineering and Regenerative Medicine Journal, vol. 4, pp. 6-20, 2011.

[30] C. Mennan, K. Wright, A. Bhattacharjee, B. Balain, J. Richardson, and S. Roberts, "Isolation and characterisation of mesenchymal stem cells from different regions of the human umbilical cord," BioMed Research International, vol. 2013, Article ID 916136, 8 pages, 2013.

[31] A. Subramanian, C. Y. Fong, A. Biswas, and A. Bongso, "Comparative characterization of cells from the various compartments of the human umbilical cord shows that the wharton's jelly compartment provides the best source of clinically utilizable mesenchymal stem cells," PLoS One, vol. 10, no. 6, article e0127992, 2015.
[32] T. Montemurro, G. Andriolo, E. Montelatici et al., "Differentiation and migration properties of human foetal umbilical cord perivascular cells: potential for lung repair," Journal of Cellular and Molecular Medicine, vol. 15, no. 4, pp. 796-808, 2011.

[33] S.-H. Hong, L. Maghen, S. Kenigsberg et al., "Ontogeny of human umbilical cord perivascular cells: molecular and fate potential changes during gestation," Stem Cells and Development, vol. 22, no. 17, pp. 2425-2439, 2013.

[34] S. L. Etheridge, G. J. Spencer, D. J. Heath, and P. G. Genever, "Expression profiling and functional analysis of Wnt signaling mechanisms in mesenchymal stem cells," Stem Cells, vol. 22, no. 5, pp. 849-860, 2004.

[35] O. O. Udartseva, E. R. Andreeva, and L. B. Buravkova, "Wnt-associated gene expression in human mesenchymal stromal cells under hypoxic stress," Doklady Biochemistry and Biophysics, vol. 465, no. 1, pp. 354-357, 2015.

[36] A. K. Batsali, C. Pontikoglou, D. Koutroulakis et al., "Differential expression of cell cycle and Wnt pathway-related genes accounts for differences in the growth and differentiation potential of Wharton's jelly and bone marrow-derived mesenchymal stem cells," Stem Cell Research \& Therapy, vol. 8, no. 1, p. 102, 2017.

[37] M. J. Nemeth, L. Topol, S. M. Anderson, Y. Yang, and D. M. Bodine, "Wnt5a inhibits canonical Wnt signaling in hematopoietic stem cells and enhances repopulation," Proceedings of the National Academy of Sciences of the United States of America, vol. 104, no. 39, pp. 15436-15441, 2007.

[38] A. J. Mikels and R. Nusse, "Purified Wnt5a protein activates or inhibits beta-catenin-tcf signaling depending on receptor context,” PLoS Biology, vol. 4, no. 4, article e115, 2006.

[39] R. van Amerongen, A. Mikels, and R. Nusse, "Alternative Wnt signaling is initiated by distinct receptors," Science Signaling, vol. 1, no. 35, article re9, 2008.

[40] G. Liu, A. Bafico, and S. A. Aaronson, "The mechanism of endogenous receptor activation functionally distinguishes prototype canonical and noncanonical Wnts," Molecular and Cellular Biology, vol. 25, no. 9, pp. 3475-3482, 2005. 

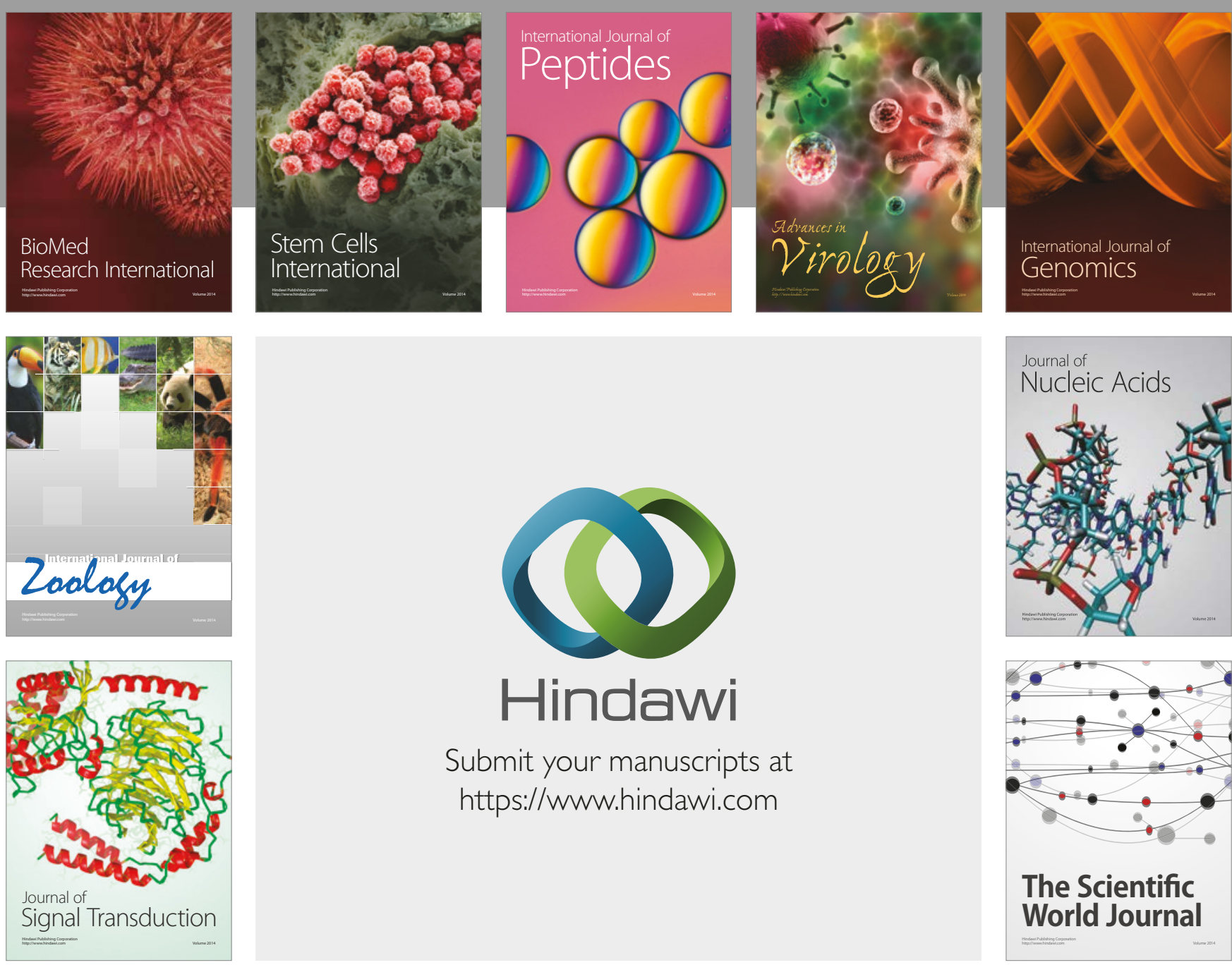

Submit your manuscripts at

https://www.hindawi.com
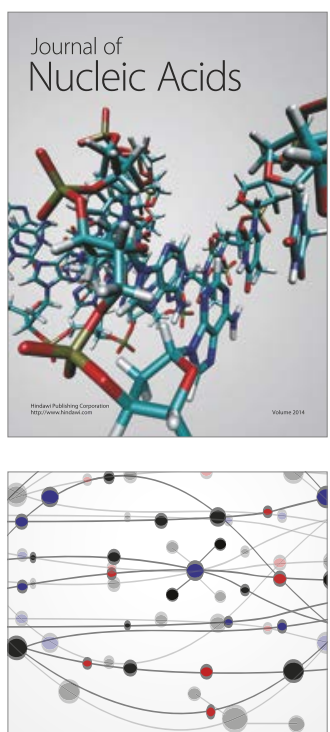

The Scientific World Journal

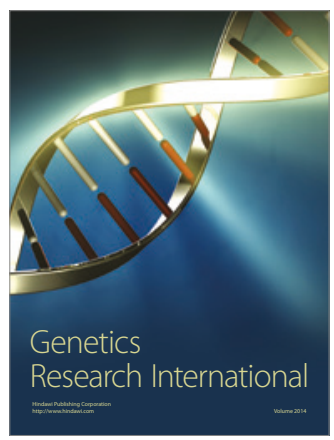

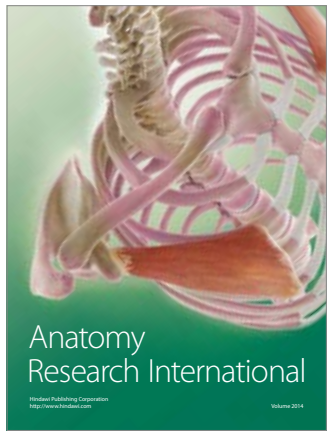

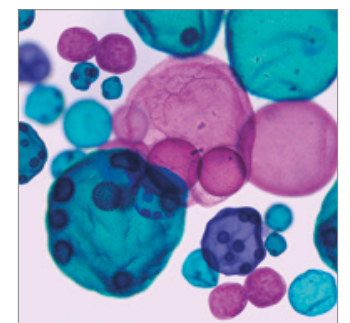

International Journal of Microbiology
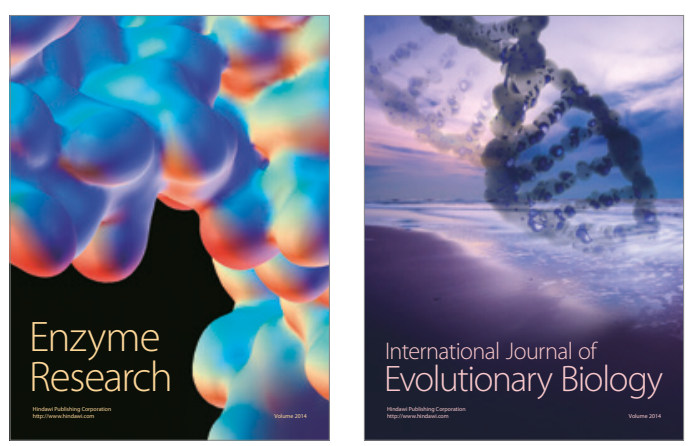
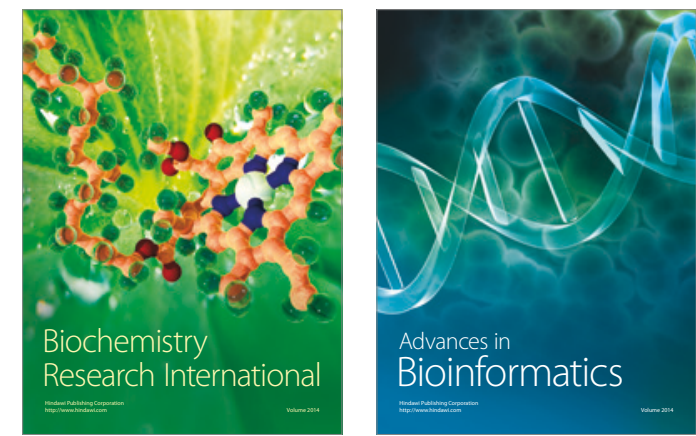

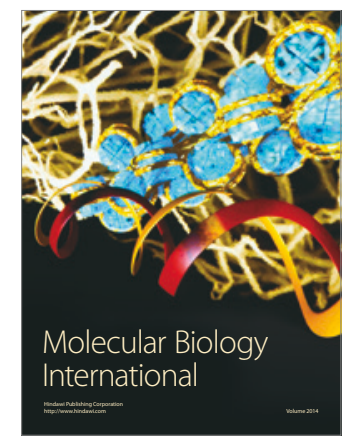

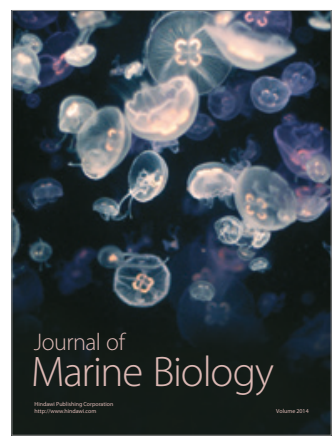

